\title{
Study of Absorption Spectra, Fluorescence and Energy Levels of Fluorescein Dye
}

\author{
Afrah M. AL Hussainey $^{\mathrm{a}} \quad$ Salar Hussein Ibrahem $^{\mathrm{b}} \quad$ Talib M. Abbas ALShafie $^{\mathrm{c}}$ \\ ${ }^{a}$ Department of Physics, college of Science, University of Babylon,Babil, Iraq. \\ ${ }^{b, c}$ Department of Physics, Education for pure sciences, Universityof Babylon, Babil, Iraq \\ afrahalsherefy@yahoo.com Dr.Talib_mohsen@yahoo.com
}

Submission date:- 22/1/2018 Acceptance date:- 29/1/2018 Publication date:- 20/9/2018

Keywords: Fluorescein dye, Absorbance, Fluorescence, Dye Laser.

\begin{abstract}
The absorption and fluorine spectra of dissolved fluorescein solution in ethanol and different concentrations were studied. The optical properties(absorbance, permeability, linear absorption and refractive index, as well as time and fluoridation)were calculated. The results showed that a decrease in concentration leads to a decrease in absorption values and an increase in permeability values. This results in a decrease in the values of the absorption coefficient and refractive index. This is in accordance with Lambert's law. Also, the values of the fluorine are lower. The top of the emission spectrum is transferred to the longer wave lengths. The temporal age of fluoride decreases and the quantitative output increases, thus using this dye as an effective laser medium in liquid state lasers. The infrared spectroscopy of the dye was also studied using the (Gaussian 9) program.
\end{abstract}

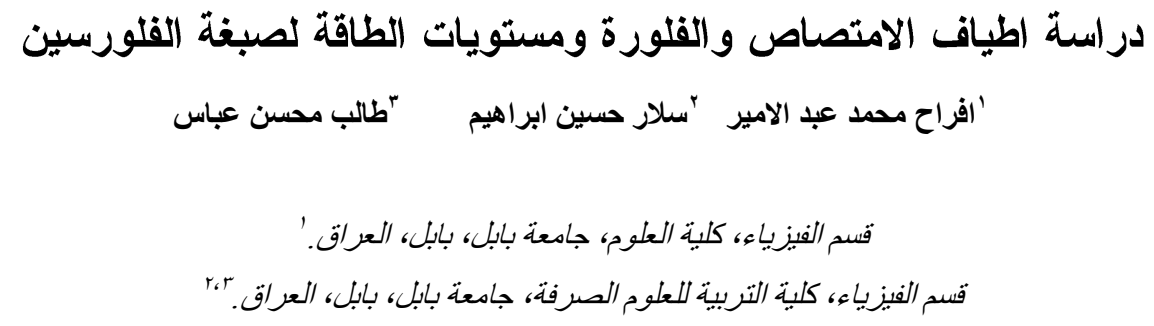

afrahalsherefy@yahoo.com Dr.Talib_mohsen@yahoo.com 


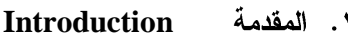

ان الصبغة العضوية هي مركب هيدروكاربوني غير مشبع ، ذات تركيب معقد نوعا ما وذلك لاحتو ائها على سلسلة من ذرات الكاربون التي ترتبط بأواصر مفردة ومزدوجة بشكل متعاقب و التي يطلق عليها نظام الكروموفور (Chromophore system) [1,2] ويتمايز

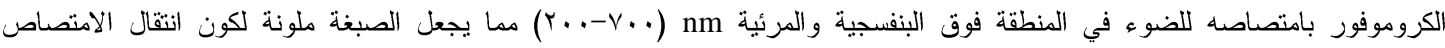

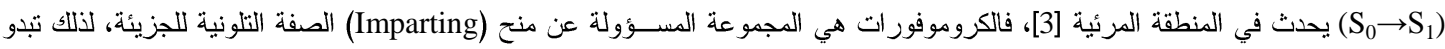

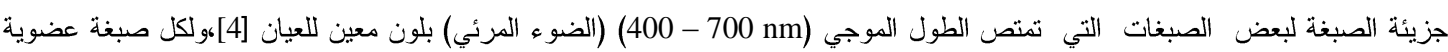

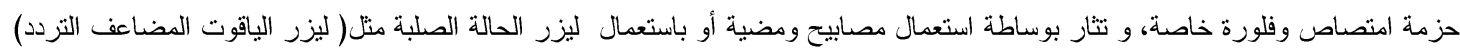
او ليزر الحالة الغازية مثل ليزر ايون الاركون او ليزر النتروجين [2,5].

The Theoretical Part r. الجزء النظري Linear optical properties ب. الخصائص البصرية الخطية إن التفاعل الحاصل بين طبيعة وتوزيع الثحنات داخل المادة (الاكترونية الجزيئية والايونية) والأشعة الكهرومغناطيسية الساقطة على المادة

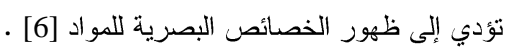
إن عددا من العمليات يمكن أن يحدث عند سقوط شعاع كهرومغناطيسي على المادة وتفاعلهما معاً , حيث ان جزء من الاتشعاع الضوئي

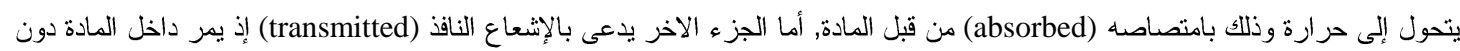

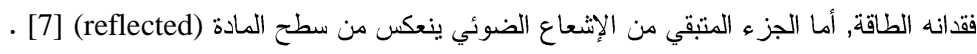
للحصول على معلومات عن التركيب الداخلي للمادة وطبيعة اواصرها يجب معرفة النفاذية والامتصاصية والانعكاسية للشعاع

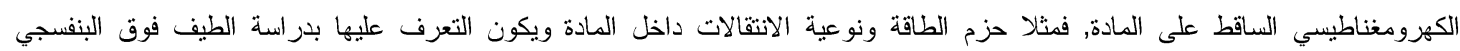
(8), أما لمعرفة مجال التطبيقات العملية التي تستخدم فيها المو اد يجب دراسة الطيف المرئي(Visible) , (Ultraviolet)

\section{Absorbance (A) الامتصاصية 1.1.2}

إن الكمية الرياضية التي تربط كثافة الجسيمات (التركيز) في العينة وسك العينة (طول المسار البصري) هي الامتصاصية (A) أو الكثافة

البصرية (Optical Density).

$A=\log \left(I_{\mathrm{o}} / I\right)$

حيث I : هي شدة الضوء عند طول الموجة ح الذي يمر خلال العينة ( شدة الـلضوء الــنافذ)، و Io : هي شدة الضوء قبل دخوله إلى العينة أو شدة الضوء الساقط.

يسبب امتصاص المادة للأشعة الساقطة نشاطاً الكترونياً قد يؤدي إلى تفكك جزيئاتها إذا كانت قيمة الطاقة الممتصة أكبر من قيمة تفكك

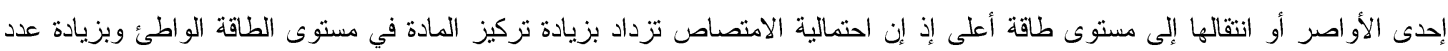
فوتونات الأشعة الساقطة [9]. إن احتمالية امتصاصية الفوتون تتناسب طردياً مع تركيز الجزيئات الممتصة في العينة وسمك الأنموذج (طول

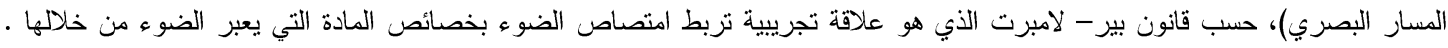
وينص القانون أن عدد الجزيئات الماصة في الماده تتتاسب طرديا مع جزء الإشعاع الممتص المار من خلالها فاذا مرت الإثعة في محلول معين

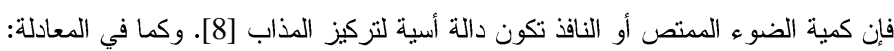
$I=I_{o} e^{-\alpha o p C m \mathrm{~L}}$

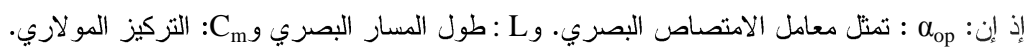
ويمكن كتابة المعادلة بالثكل الاتي:

$\ln \mathrm{I}_{\mathrm{o}} / \mathrm{I}=\alpha_{\mathrm{op}} \mathrm{C}_{\mathrm{m}} \mathrm{L}=\mathrm{A}$

إذ يمكن تطبيق قانون بير- لامبرت ( Lambert - Beer Law ) في مخـتلف الـــناطق الطيفية كالأشعة الفوق البنفسجية والمرئية وغيرها

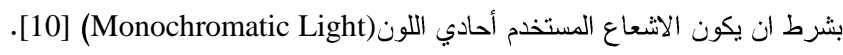

\section{Absorption Coefficient \\ (a) T.1.r}

يمكن تعريف معامل الامتصاص على أنه نـــسبة التـناقص في فيض طــاقة الاثــعاع الـساقط بالنسبة لوحدة المسافة باتجاه

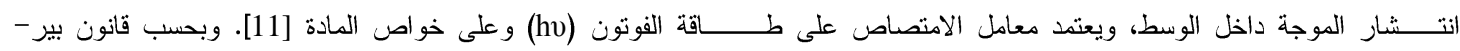

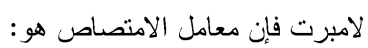


$\log \left(\mathrm{I}_{\mathrm{o}} / \mathrm{I}\right)=2.303 \mathrm{~A}=\alpha_{\mathrm{o}} \mathrm{d}$

$\alpha_{\mathrm{o}}=2.303 \mathrm{~A} / \mathrm{d}$

Transmittance (T) النفاذية

تعرف النفاذية للوسط (transmittance -T) على أنها"النسبة المئوية لثدة الضوء النافذ (II) إلى شدة الضوء الساقط (II) "، أو أنها " طاقة

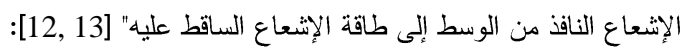

$\mathrm{T}=\left(\mathrm{I} / \mathrm{I}_{\mathrm{o}}\right)$

و اسنتادا إلى قانون بير- لامبرت فان النفاذية تتتاقص كلما زاد التركيز المولاري C وطول المسار البصري L الذي يمر خلاله الضوء [14]. أما نفاذية الوسط ترتبط بامتصاصية المحلول (Absorbance -A) بالعلاقة الآتية:

$A=-\log (1 / T)=-\log \left(I / I_{0}=\log \left(I_{0} / I\right)\right.$

ومن هذه العلاقة نلاحظ إن النفاذية T تزداد كلما قلت امتصاصية الوسط A.

Refractive Index (n) الآكسار

ينتقل الضوء بجميع أطو اله الموجية بأقصى سرعة له في الفراغ وهي كمية ثابتة وتقل هذه القيمة في أب وسط أخر , و إنها تتغير في الخي الاوساط المادية باختلاف الاطو ال الموجية.

وتعرف النسبة بين سرعة الضوء في الفراغ إلى سرعته في أي وسط معين لطول موجة معينة بمعامل الانكسار الوسط لتلك الموجة [12]. $\mathrm{n}=\mathrm{c} / \mathrm{v}$

$$
\text { حيثc: سرعة الضوء في الفراغ و : سرعة الضوء في الاوساط المادية . }
$$

إن معامل الانكسار غير ثابت ويعتمد على طول الموجة الكهرومغناطيسيّة. فضلا عن بعض المواد التي يختلف فيها معامل الانكسار على

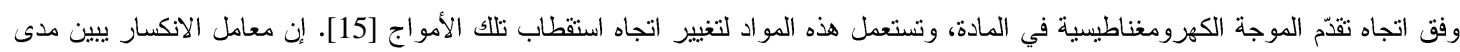

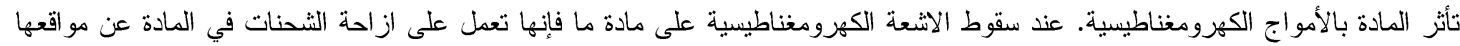

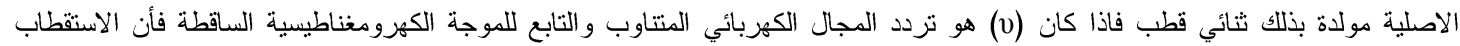
الكهربائي للجزيئة سوف يتذبذب بنفس التزدد(v), وإن جزء من طاقة الموجة الساقطة سوف يتحول إلى طاقة اهتز ازية لثنائي القطب الكهربائي المتولد, وبذلك تتقص سعة الموجة الساقطة, وعلى فرض أن الفقدان في الطاقة يسبب تذبذب الثنائيات بشكل قليل, إلا أن فعل التأخير الحاصل في

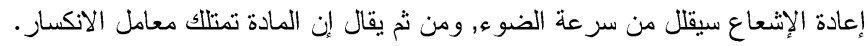

ومن هنا يتضح إن الاستقطاب في المادة بفعل سقوط الاشعة الكهرومغناطيسية عليها مقياس لمعامل الانكسار لهذه المادة , فكلما كان

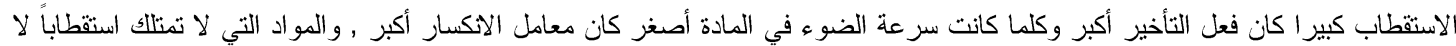

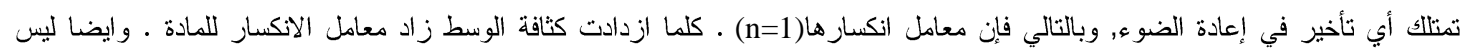

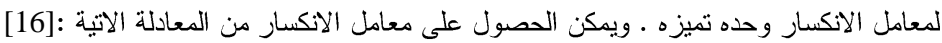
$n_{o}=\frac{1}{T}\left[\left(\frac{1}{T^{2}}-1\right)\right]^{1 / 2}$

\section{Quantum Efficiency الكفاءة الكمية}

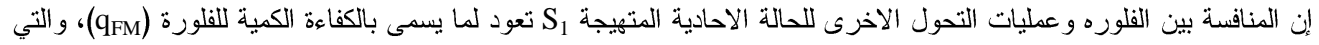

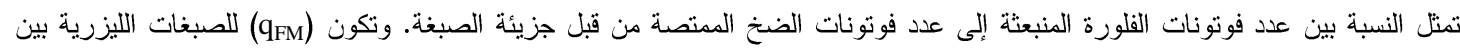

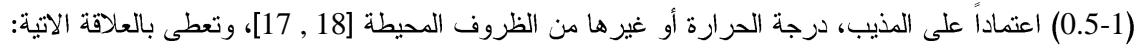

$q_{F M}=\int_{0}^{\infty} F(v) d v$ 
عندما تمنص المادة الاشعاع الكهرومغناطيسي فأنها تثار وتزداد طاقتها, ويمكن لهذه المادة المثارة ان تبث فوتونات مختلفة الطاقة حتى تصل

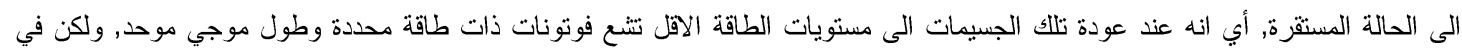

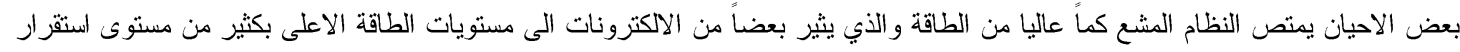

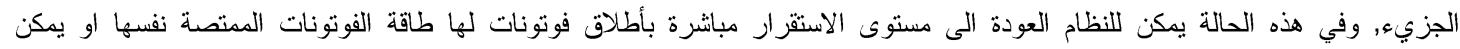

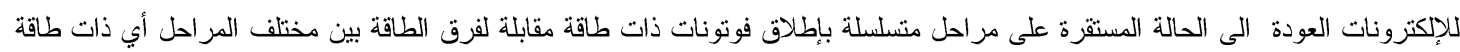

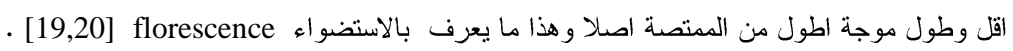

(Gaussian 09) برنامج r.r

برنامج (Gaussian 09) هو من بين سلسلة من البرامج حملت الاسم نفسة لموديلات مختلفة. وهو أحد برامج التزكيب الإلكتروني,

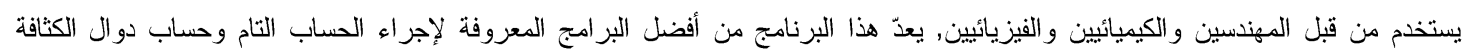

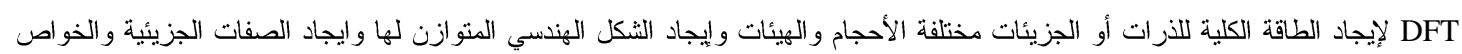

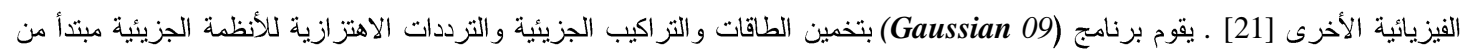

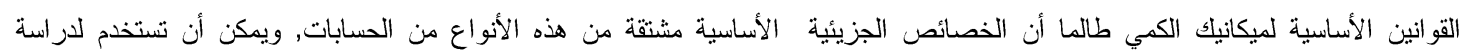
الجزيئات و التفاعلات تحت مدى واسع من الظروف, مشتمل على المكونات المستقرة و المركبات التي من الصعب أو المستحيل ملاحظتها تجريبيا منل وسطيات التفاعل ونز اكب الحالة الانتقالية.

\section{r. (الجزء العملي \\ ب ا.أجزة المستخدمة}

سجل طيف الامتصاص باستخدام مطياف ثنائي الحزمة (UV-Visible- Spectrophotometer) ويغطي هذا المطياف منطقة واسعة من الطيف الكهرومغناطيسي من المنطقة فوق البنفسية حتى منطقة تحت الحمراء القريبة ، ويشتنل الجهاز على مصدرين للتهييج هما

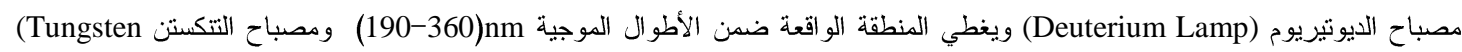

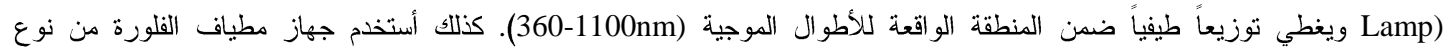
F96PRO

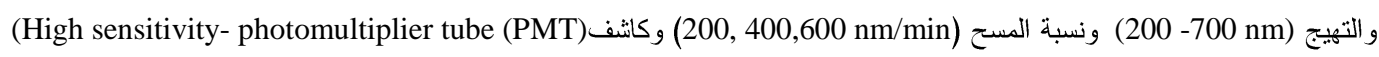

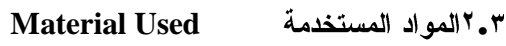 \\ Fluorescein Dye أ- صبغة الفلورسين}

تكون هذه الصبغة بشكل مسحوق بلوري Orange وتبدو هذه الصبغة بشكل محلول مصفر وند إذابتها في المحاليل القاعدية

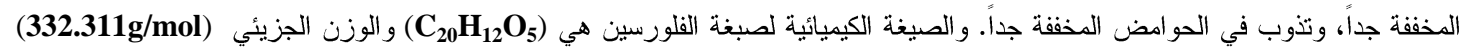
و الثكل (1) يوضح التركيب الجزيئي للفلورسين.

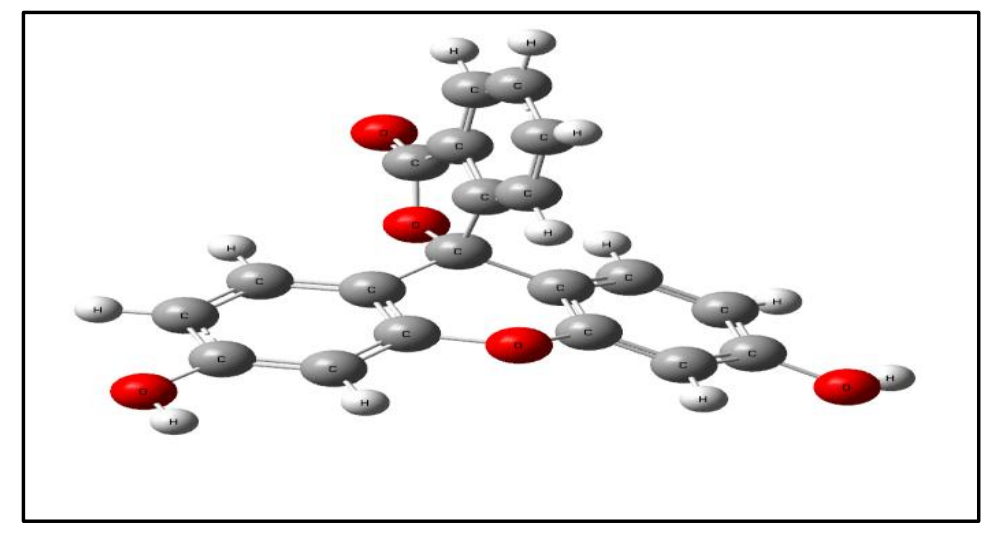

الثكل (1) الثركيب الجزيئي لصبغة (الفلورسين 


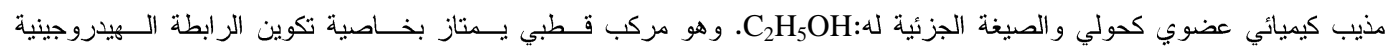

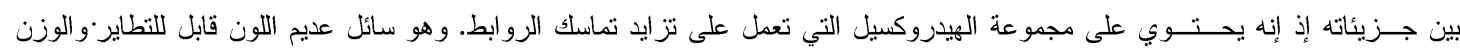

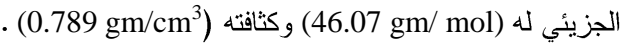

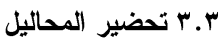

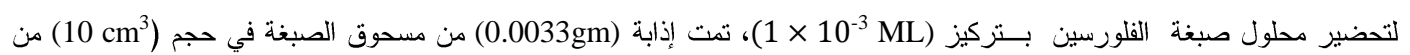

مذيب الايثانول (Ethanol) الذي أستخدم في البحث وفقاً للعلاقة:

$W m=\frac{C \times V \times M \cdot W}{1000}$

$$
\begin{aligned}
& \text { gm وز وزن الصبغة اللازم للحصول على التركيز المطلوب بوحدة: } \\
& \text { ML التركيز المراد تحضيره بوحدة: C } \\
& \text { cm³ اللازم اضافته إلى المادة. V حجم المذيب. V } \\
& \text { gm / mol الوزن الجزيئي للصبغة المستخدمة : : M.W }
\end{aligned}
$$

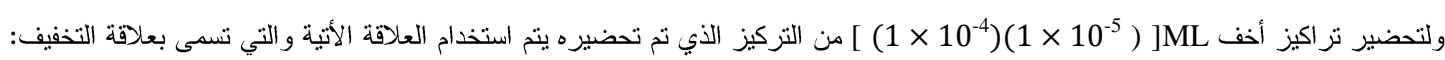
$\mathrm{C}_{1} \mathrm{~V}_{1}=\mathrm{C}_{2} \mathrm{~V}_{2}$

$$
\begin{aligned}
& \text { حيث : } \\
& \text { - } \\
& \text { C التركيز الثاني (الأخف). }
\end{aligned}
$$

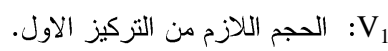

$$
\begin{aligned}
& \text { : الحجم اللازم اضافته للتزكيز الاول للحصول على التركيز الثاني. }
\end{aligned}
$$

Calculation and Results و. الحسابات والنتائج

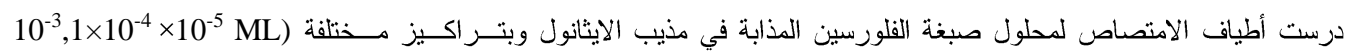
(1×1 1) باستخدام مطياف الأشعة المرئية والفوق البنفجية (UV -Visible Spectrophotometer) كما هو موضح في الثكل (2) والجدول 


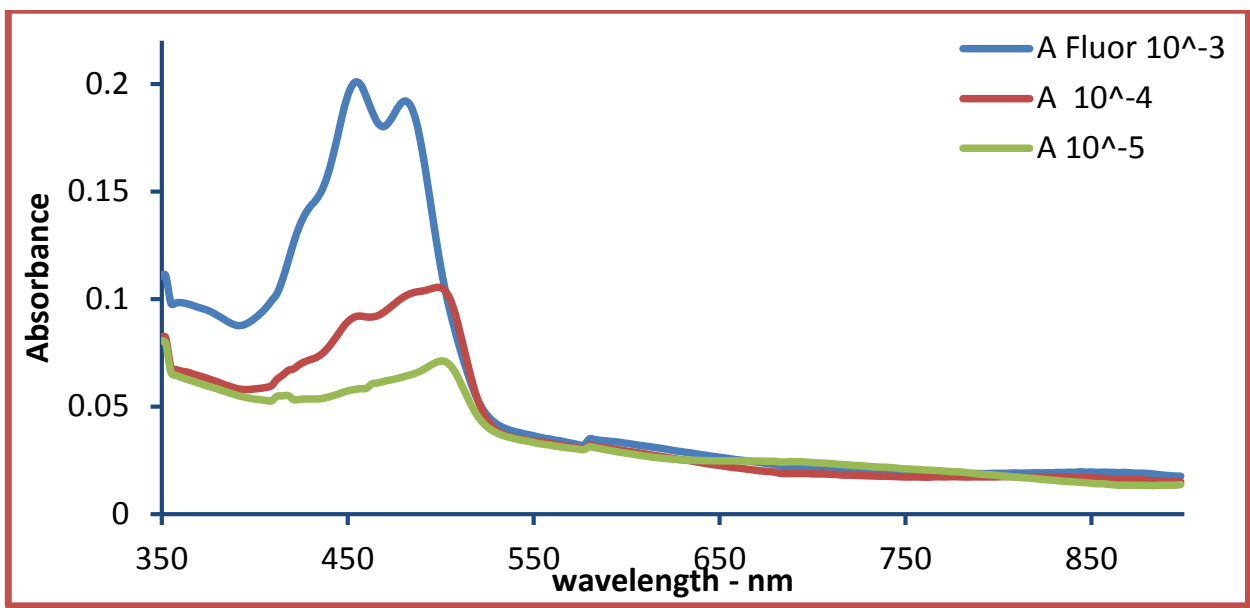

الثكل (2) منحنيات طيف الامتصاصية لمحلول صبغة الفلورسين المذابة في الايثانول وبتر اكيز مختلفة.

جدول (1) الامتصاصية عند الأطوال الموجية العظى للصبغة الفلورسين المذابة في الايثانول وبتر اكيز مختلفة

\begin{tabular}{||c||c|c||}
\hline $\mathbf{C}(\mathbf{M L})$ & $\boldsymbol{\lambda}_{\text {max }}(\mathbf{n m})$ & $\mathbf{A}$ \\
\hline \hline $1 \times 10^{-3}$ & 451 & 0.1968 \\
\hline \hline $1 \times 10^{-4}$ & 493 & 0.1042 \\
\hline \hline $1 \times 10^{-5}$ & 496 & 0.0698 \\
\hline
\end{tabular}

نلاحظ من الجدول ان شدة الامتصاص تزداد بزيادة التركيز وهذا ينطبق مع معادلة بير لامبرت للامتصاص و الزيادة في التركيز المولازي

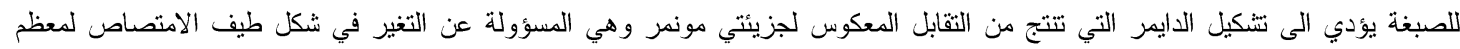

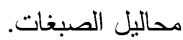

ومن نتائج أطياف الامتصاص أمكن الحصول على أطياف النفاذية (Transmission) وكما هو موضح بالشكل (3) والجدول (2).

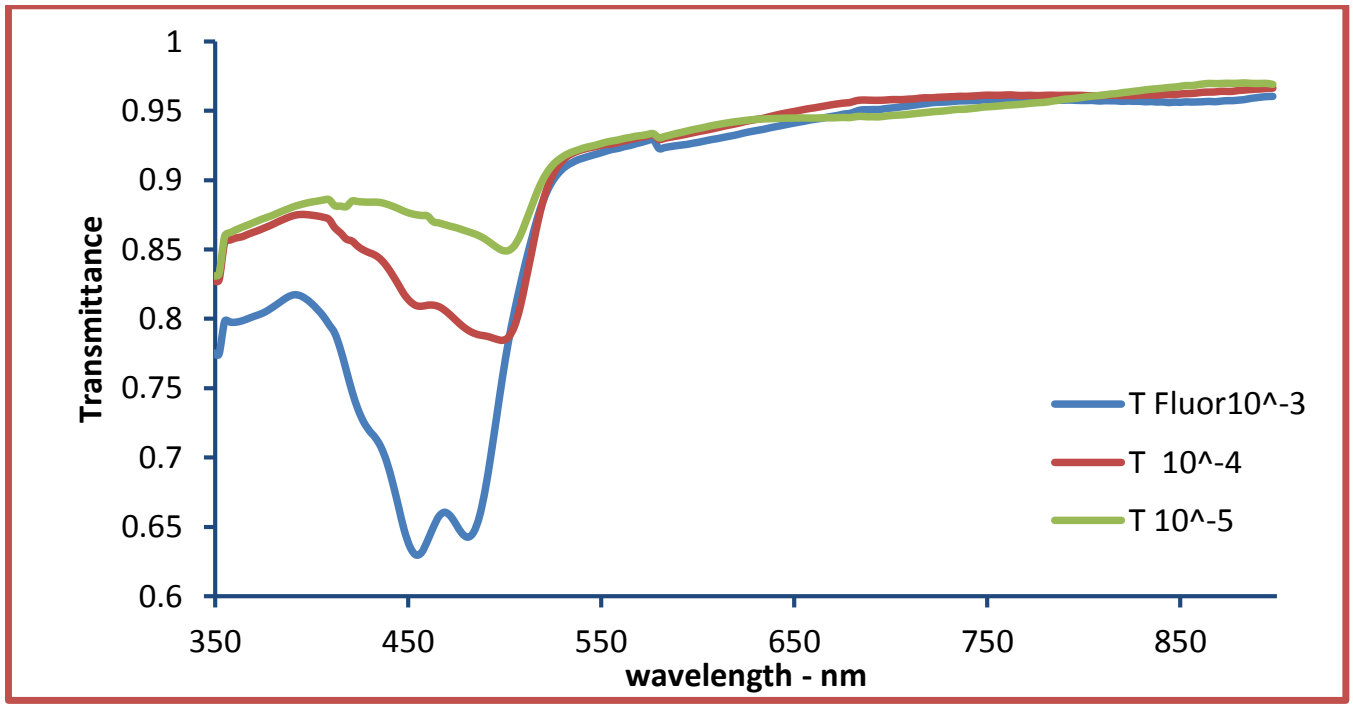

الثكل (3) منحنيات طيف النفاذية لمحلول صبغة الفلورسين المذابة في الايثانول وبتر اكيز مختلفة. 
جدول (2) النفاذية عند الأطوال الموجية العظى للصبغة الفلورسين المذابة في الايثانول وبتر اكيز مختلفة

\begin{tabular}{||c||c|c|}
\hline $\mathbf{C}(\mathbf{M L})$ & $\lambda_{\max }(\mathbf{n m})$ & $\mathbf{T}$ \\
\hline \hline $1 \times 10^{-3}$ & 451 & 0.6355 \\
\hline \hline $1 \times 10^{-4}$ & 493 & 0.7865 \\
\hline \hline $1 \times 10^{-5}$ & 496 & 0.8514 \\
\hline
\end{tabular}

وبعد الحصول على نتائج الامتصاصية والنفاذية حسب معامل الامتصاص (a) (م) ومعامل الانكسار (no ) الخطيين للنماذج المحضرة المعادلتين (5) و (9) وكما هو موضح في الجدول (3).

جدول (3) قيم معامل الاككسار الخطي والامتصاص الخطى لمحلول صبغة الفلورسين المذابة في الإيثانول وبتر اكيز المختلفة

\begin{tabular}{|c||c||c|}
\hline $\mathbf{C}(\mathbf{M L})$ & $\boldsymbol{\alpha o}(\mathbf{c m})^{-1}$ & $\mathbf{n}_{\mathbf{0}}$ \\
\hline \hline $1 \times 10^{-3}$ & 0.17 & 1.85 \\
\hline \hline $1 \times 10^{-4}$ & 0.16 & 1.80 \\
\hline \hline $1 \times 10^{-5}$ & 0.15 & 1.76 \\
\hline
\end{tabular}

قيست أطياف الفلورة للنماذج المحضرة لمحلول صبغة الفلورسين المذابة في الايثانول وبتراكيز مختلفة باستخدام مطياف الفلورة (Spectrofluorometer)

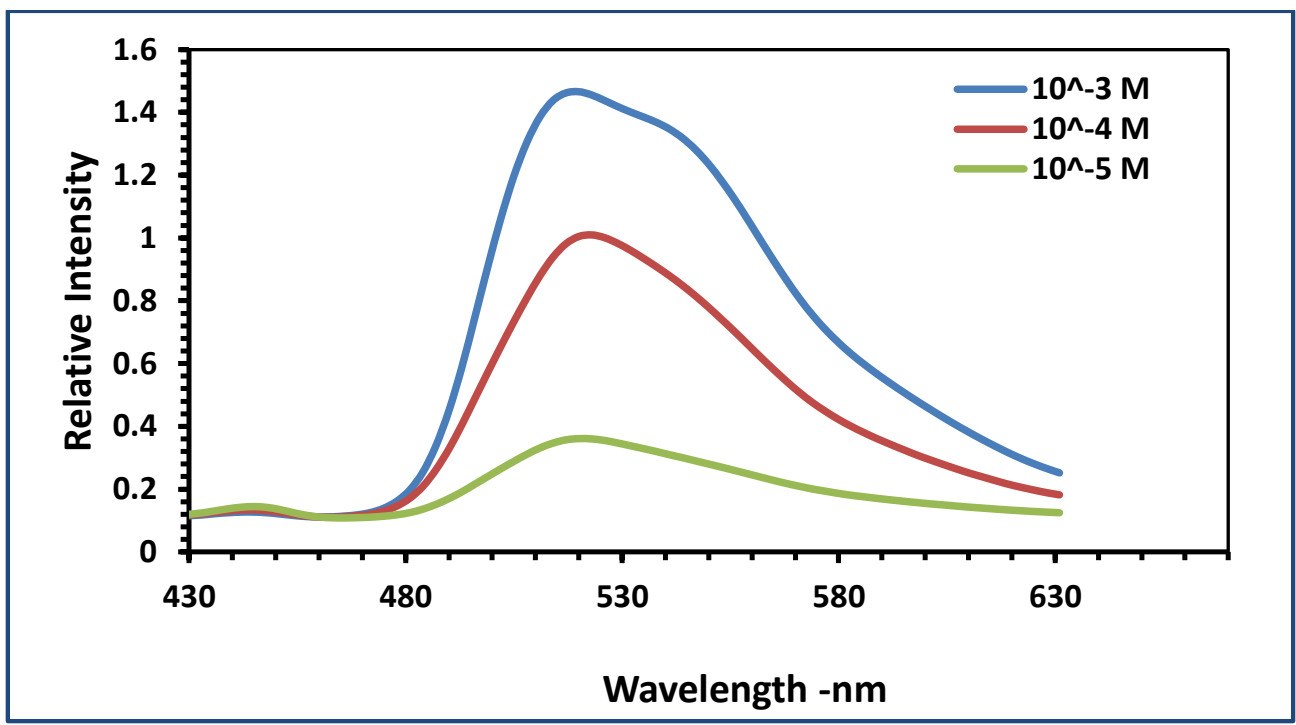

الثكل (4) منحنيات طيف الفلورة لمحلول صبغة الفلورسين المذابة في الايثانول وبتر اكـيز مختلفة. 
جدول (4) قيم شدة الفلورة عند الأطوال الموجية العظى لمحلول صبغة الفلورسين المذابة في الايثانول وبتر اكيز مختلفة .

\begin{tabular}{|c||c||c||}
\hline $\mathbf{C}(\mathbf{M L})$ & $\lambda_{\max }(\mathbf{n m})$ & Relative Intensity \\
\hline \hline $1 \times 10^{-3}$ & 523 & 1.4655 \\
\hline \hline $1 \times 10^{-4}$ & 517 & 0.9815 \\
\hline \hline $1 \times 10^{-5}$ & 514 & 0.3465 \\
\hline
\end{tabular}

ومن نتائج أطياف الفلورة أمكن حساب العمر الزمني للفلورة (fluorescence lifetime) (

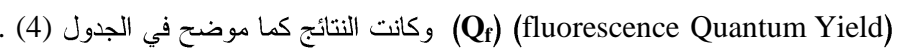

جدول (4) العمر الزمني و النتاج الكمي للفلورة لمحلول صبغة الفلورسين المذابة في الايثانول وبتر اكيز مختلفة.

\begin{tabular}{|c||c|c||}
\hline $\mathbf{C}(\mathbf{M L})$ & $\tau_{\mathbf{f}}(\mathbf{n s})$ & $\mathbf{Q}_{\mathbf{f}}$ \\
\hline \hline $1 \times 10^{-3}$ & 0.31 & 0.60 \\
\hline \hline $1 \times 10^{-4}$ & 0.30 & 0.93 \\
\hline \hline $1 \times 10^{-5}$ & 0.19 & 0.97 \\
\hline
\end{tabular}

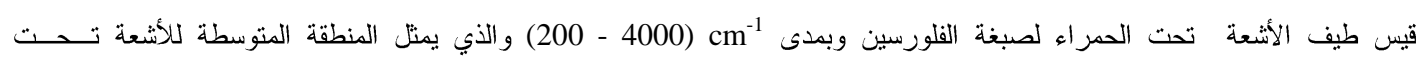

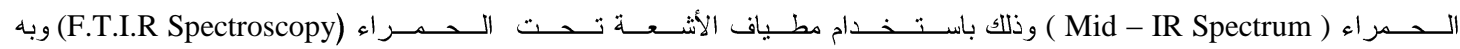
أمكن حساب معظم أنماط الاهتز از الجزيئية وشداتها لصبغة الفلورسين وكما في الثكل (5) و الجدول (5). 


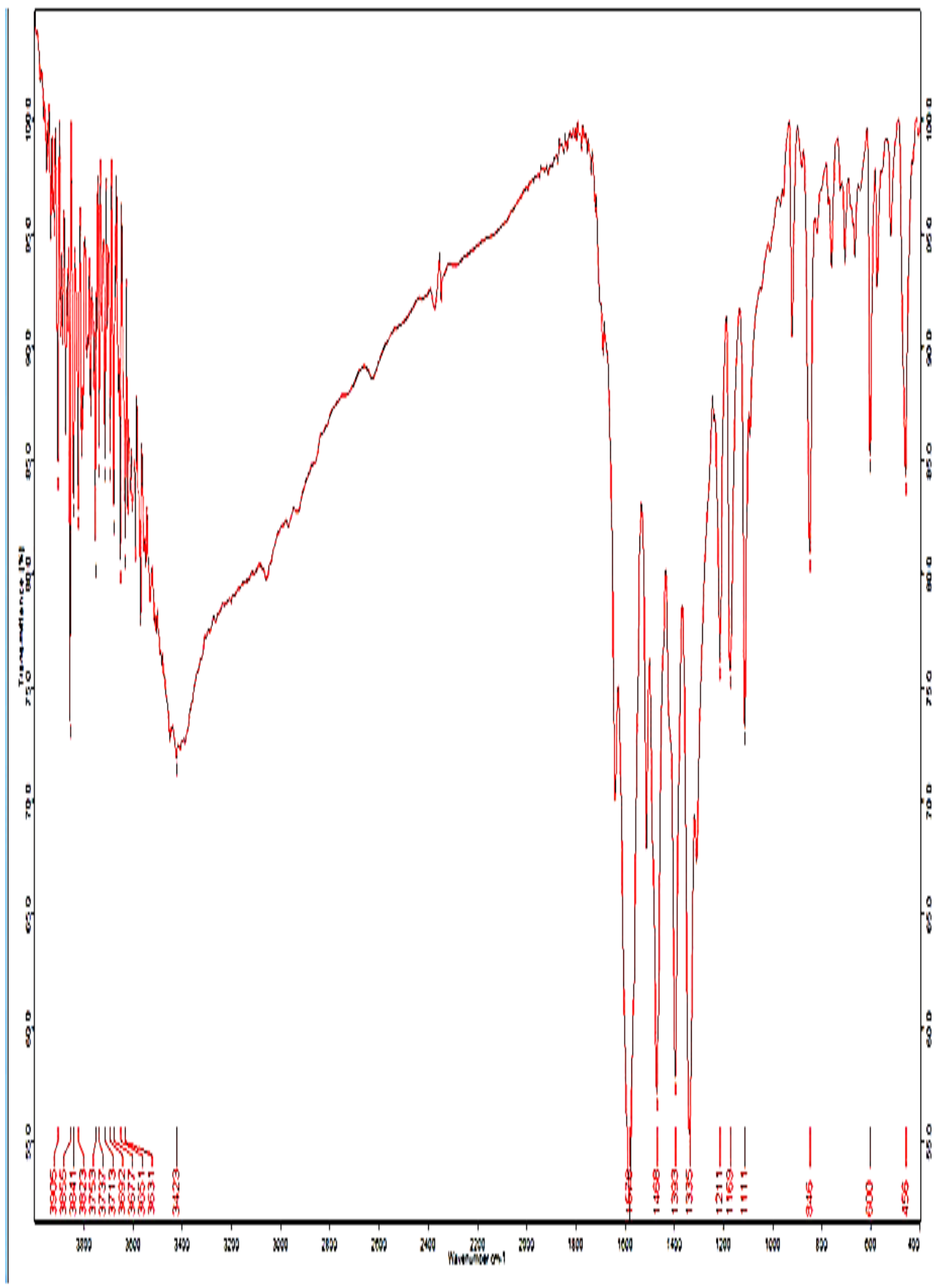

الثكل (5) طيف الاثعة تحت الحمر اء لصبغة الفلورسين والمقاس عملياً 
جدول (5) انماط الاهتزاز لجزيئة الفلورسين وشداتها المقاسة عملياً .

\begin{tabular}{|c|c|c|}
\hline Mode & $\begin{array}{l}\text { Wave No. } \\
\left(\mathrm{cm}^{-1}\right) \text { Exp. }\end{array}$ & $\begin{array}{c}\text { Intensity } \\
\text { Exp. }\end{array}$ \\
\hline $\mathbf{C}-\mathbf{H}_{\text {str }}$ & 3823 & 0.07 \\
\hline $\mathbf{C}-\mathbf{O}_{\text {ben }}$ & 3423 & 0.15 \\
\hline $\mathrm{C}-\mathbf{H}_{\text {o-o-p.def }}$ & 1570 & 0.30 \\
\hline $\mathbf{C}-\mathbf{C}_{\text {str }}$ & 1468 & 0.24 \\
\hline $\mathbf{C}-\mathbf{H}_{\text {i-o-p.def }}$ & 1393 & 0.23 \\
\hline $\mathbf{C}=\mathbf{C}_{\text {str. }}$ & 1335 & 0.25 \\
\hline $\mathbf{O}-\mathbf{H}_{\text {ben }}$ & 1211 & 0.11 \\
\hline $\mathrm{C}-\mathbf{H}_{\text {o-o-p.def }}$ & 1169 & 0.04 \\
\hline $\mathrm{C}-\mathrm{C}_{\mathrm{str}}$ & 1111 & 0.13 \\
\hline $\mathbf{C}-\mathbf{H}_{\text {ben }}$ & 846 & 0.09 \\
\hline $\mathrm{C}-\mathrm{O}_{\text {ben. }}$ & 600 & 0.07 \\
\hline $\mathbf{C}-\mathbf{H}_{\text {ben. }}$ & 456 & 0.075 \\
\hline
\end{tabular}

نظرياً يمكن الحصول على أنماط الاهتزاز وشداتها باستخدام برنامج (Gaussian 09) والذي يقيس لأنماط الاهتزاز بالحالة الغازية الاحادية

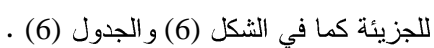

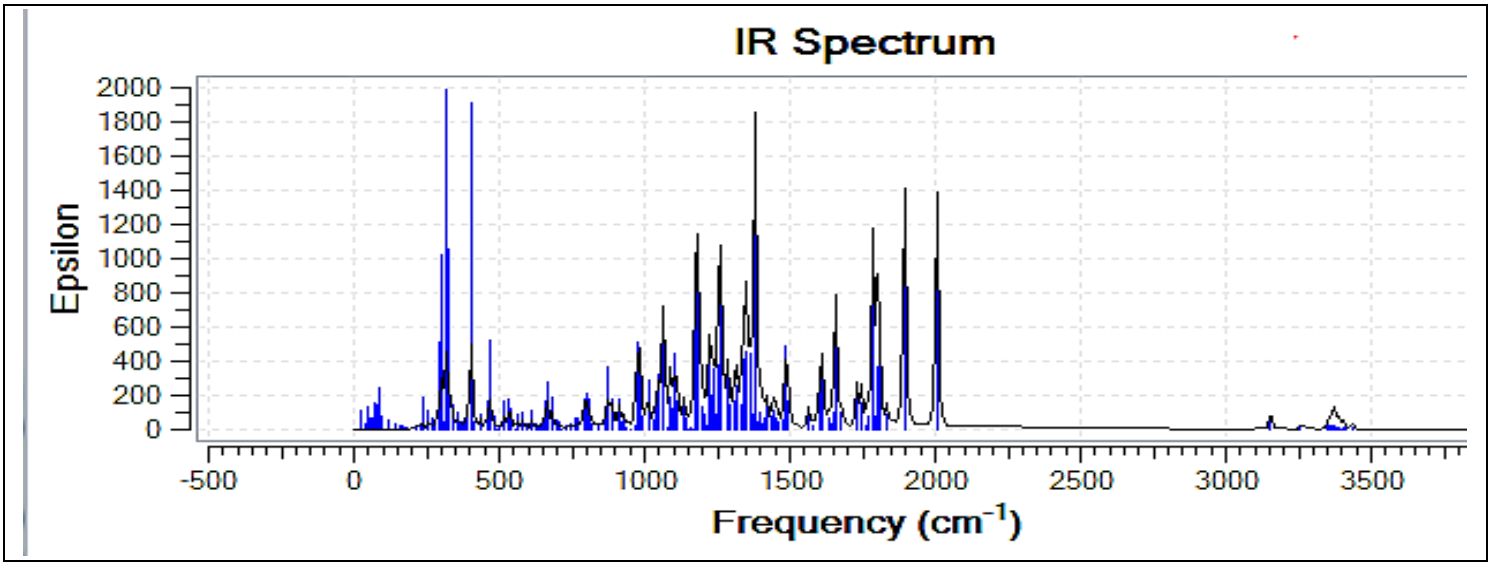

الثكل (6) طيف الاشعة تحت الحمر اء لصبغة الفلورسين والمقاس نظرياً. 
جلول (6) انماط الاهتزاز لجزيئة الفلورسين وشداتها المقاسة نظرياً

\begin{tabular}{|c|c|c|}
\hline Mode & $\begin{array}{l}\text { Wave No. } \\
\left(\mathrm{cm}^{-1}\right) \text { Theo. }\end{array}$ & $\begin{array}{c}\text { Intensity } \\
\text { Theo. }\end{array}$ \\
\hline $\mathrm{C}-\mathrm{H}_{\mathrm{str}}$ & 3438 & 0.07 \\
\hline $\mathrm{C}-\mathrm{O}_{\text {ben }}$ & 3408 & 0.14 \\
\hline $\mathrm{C}-\mathrm{H}_{\text {o-o-p.def }}$ & 1564 & 0.37 \\
\hline $\mathrm{C}-\mathrm{C}_{\mathrm{str}}$ & 1479 & 0.19 \\
\hline $\mathrm{C}-\mathrm{H}_{\text {i-o-p.def }}$ & 1396 & 0.21 \\
\hline $\mathrm{C}=\mathrm{C}_{\text {str. }}$ & 1345 & 0.30 \\
\hline $\mathrm{O}-\mathrm{H}_{\text {ben }}$ & 1218 & 0.11 \\
\hline $\mathrm{C}-\mathrm{H}_{\text {o-o-p.def }}$ & 1170 & 0.08 \\
\hline $\bar{C}-\mathrm{C}_{\text {str }}$ & 1139 & 0.17 \\
\hline $\mathrm{C}-\mathrm{H}_{\text {ben }}$ & 866 & 0.10 \\
\hline$\overline{\mathrm{C}}-\mathrm{O}_{\text {ben. }}$. & 630 & 0.05 \\
\hline$\overline{\mathrm{C}}-\mathrm{H}_{\text {ben. }}$. & 491 & 0.07 \\
\hline
\end{tabular}

لقد قيست الثدة (Intensity) لهذه الأنماط الاهتزازية نظرياً، باستخدام برنامج (Gaussian 09)، فالانتقال الذي يشمل تغيراً كبيراً في

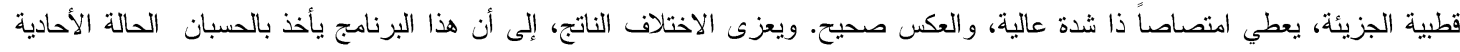

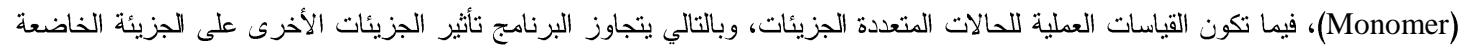

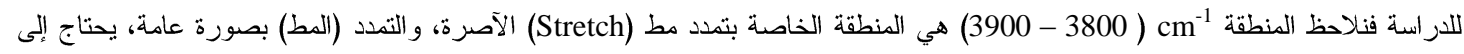

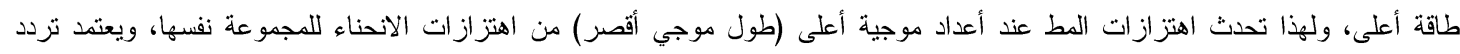

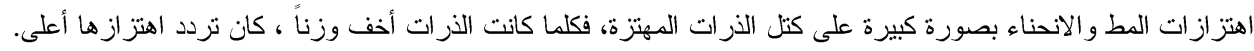

•.

نلاحظ من در اسة الخصائص الطيفية لصبغة الفلورسين المذابة في الإيثانول ان نقصان التركيز يؤدي الى ظهور اطياف ذات قمم تقل شدتها

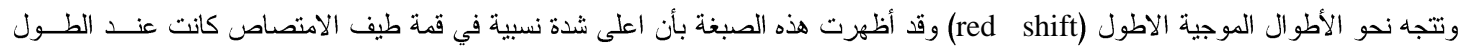
الموجي (484 nm) عند التركيز

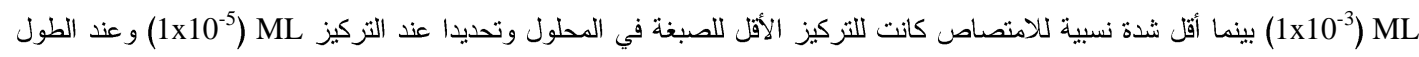
الموجي ( nm nm)، ويعزى ذلك الى زيادة عدد الجزيئات الذي يؤدي الى زيادة احتمالية الامتصاص وهذا يؤكد الامتصاص الحقيقي للصبغات

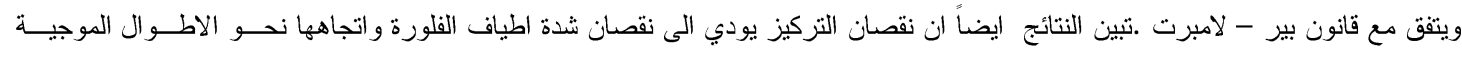

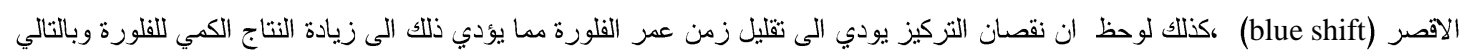
يمكن استخدام هذه الصبغة كوسط فعال في ليزرات الصبغة. كذلك درست المستويات الطاقية التذبذبية نظرياً وعمليا فقد نم الحصول على (12) نمط من انماط الاهتزاز نظريا وكانت ابرز هذه الانماط

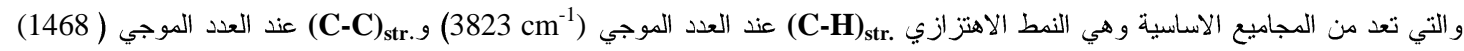

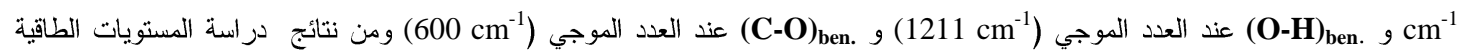
الجزيئية الاهنز ازية لاحظنا تطابقاً ملحوظاً في نتائج انماط الاهنز از المقاسة عملياً ونظرياً مع بعض الاختلاف النسبي في قيم شداتها. 


\section{CONFLICT OF INTERESTS}

\section{There are no conflicts of interest.}

[1] W.Demtroder, "Laserspectroscopy",Springer-Verlag New York, pp. 336-350, 1981.

[2] M.D.Lumb,"Lumiescence Spectroscopy", Academic Press London, 1978.

[3] Ethanan Sahar and David Treve, IEEE J. QE 13(12), pp. 692-967 , 1977.

[4] Joseph T. Verdeyen "Laser Electronics", Printice-Hall, 1986.

$$
\text { [0] سهام عفيف قندلا، "فيزياء الليزر و بعض التطبيقات العملية"، } 1987 .
$$

[6] J. Klinowski, "High-Resolution Molecular Spectroscopy Electronic Spectroscopy", University of Cambridge , 2005.

$$
\text { [7 حسن الثربيني , بتول الخياط, صبحي كمال حسون , " البصريات الفيزياوية " , جامعة بغداد \كلية التربية , ب1919. }
$$

[8] Grant, R. Fowels, " Introduction to modern optics" , holt Rinehart and Winston , 1975.

[9] J. Klinowski, "High-Resolution Molecular Spectroscopy Electronic Spectroscopy", University of Cambridge , 2005.

[10] M.Wang, Z. Lin and G. Zhai, "Optical linearity and nonlinearity of ZnSe nanocrystals embedded in epoxy resin matrix investigated by z-Scan technique", Ceramics International , 34, 1073-1076, 2008.

[11] Hollas, J.M. ,"Modern Spectroscopy" John Wiley and sons Ltd, The Atrium, Southern Cate Chionester, west Sussex PO19 8SQ, England, 2004.

[12] M . Parikh, “Absorption Spectroscopy of Organic Molecules”, Adison-Wesleg, 1974.

[13] W. Demtröder, "Atoms ,Molecules and Photons", Springer Berlin Heidelberg New York, 2010.

[14] B. P. Straughan et al. , "Spectroscopy", Chapman and Hall, London, 1976.

$$
\text { [15] ليلى محمد نجيب سليم. "الطيف" . جامعة الموصل ،1910. }
$$

[16] Y. Gun ,and H Chong, "Simple Optical Methods for Measuring Optical Nonlinearities and Rotational Viscosity In Nematic Liquid Crystals ", ISBN 978-953-307-015-5, pp.111-126, Vienna, Austria, 2009.

[17] J.B. Birks: Photophysics of Aromatic Molecular, John Wiley \& Sons, Ltd, 1970.

[18] A. Skoog Douglas: Principles of Instrument Analysis, Third ed, 1985.

[19] S. Mohamed, "The calculation of Potential energy level of semicoductor PBS using Quantum Mechanics Semiempirical method" M.Sc Thesis, Al-Mustansiriyah University, 2009.

[20] S.Hayashi ", Theoretical Study of Electronic Structure and Spectroscopy of Molecules Containing Metallic Atoms, Ph. D. Thesis , University of Paris, 2008.

[21] O.Svelte," Principles of Lasers", Plenum press, New York, 1989. 